\title{
Challenge and implications of open government-A survey of Shandong Province
}

\author{
Wei Wang1, 3, a, Yikai Liang, 2, 4, b ${ }^{\text {, Ke He }}{ }^{4, \mathrm{c}}$ and Yiming Liu ${ }^{1,3, \mathrm{~d}}$ \\ ${ }^{1}$ Shandong Computer Science Center (National Supercomputer Center in Jinan), Shandong Provincial Key \\ Laboratory of Computer Networks, Jinan, 250101, China \\ ${ }^{2}$ School of Management Science and Engineering, Shandong University of Finance and Economics, Jinan \\ 250014, China \\ ${ }^{3}$ Shandong Institute of Economy and Informatization Development, Jinan, 250014, China \\ ${ }^{4}$ School of Management, Shandong University, Jinan 250100, China \\ a'wangw@sdas.org, byikailiang@qq.com, 554241423@qq.com, diuym@sdas.org
}

Keywords: Open Government, challenge, implications, local government, China.

\begin{abstract}
Open government is of great significance for the government to establish efficient and transparent government service. Some developed countries over the world have paid great attention to open government, and developed some relevant policies and regulations about the open government. In order to further improve government transparency, China' center government also keep pace with this open trend. Therefore, using the investigation method and taking Shandong Province as an example, we organized open assessment working group to assess the Shandong provincial government departments and the municipal government in 2016 and analyzed the result. Then, we find the challenge existing in the present stage, and put forward some implications for improvement.
\end{abstract}

\section{Introduction}

Public affairs mainly refers to the work of government public, the work for the government planning or preparing for the classification of the public, the work content and process to the public, any citizen can carry out query and supervision through specific ways, such as open data, open network and so on [1]. Openness of government data and affairs play an important role in promoting administration according to law, promoting the transformation of government functions, improving administrative efficiency, preventing and controlling corruption [2]. In the course of more than 20 years' practice, the openness of government affairs in our country has not only accumulated rich experience, but also appeared the situation of lower legislative level and unreasonable mechanism, which needs further improvement [3]. At present, the Shandong provincial government has responded positively to the call of the state, and has made great improvements in government work, but there are still many problems. At the same time, there are many deficiencies in the research of the open government, and lack of targeted research. This paper takes Shandong Province (SD) as an example, evaluate and analysis the open government of SD in 2016, to explore the present situation of Shandong government, and found the public problems, put forward some implicationss for improvement.It will bring beneficial enlightenment for the government to effectively promote the open government.

\section{Research Methodology}

The purpose of this study is to explore the current situation of the openness of government affairs in SD and find out the existing problems. Using survey method to collect can get some information 
that difficult to obtain from direct observation, not restricted by time and space, and has high efficiency, can get a lot of data in a short period of time, at the same time to promote the work of relevant units itself through investigation, and it is widely used in the research on the current problem. Therefore, this article adopts the survey method to evaluate and analyze the government affairs of the relevant departments in SD in 2016.

The assessment target is 42 provincial government departments (including constituent sectors, departments that directly under the ad hoc agencies, institutions and departments directly under the Administration) and 17 municipal governments.

The evaluation index includes four first level indicators: "Openness of administrative power operation", "Information openness in key fields", "Government involvement and response concerns" and "Capacity building of open government". The corresponding weights were $30 \%, 25 \%$, 25\%, and 20\%. In addition, the four first level indexes contains 16 second level indexes, covers six aspects of Public Affairs (the management of public services, public policy implementation, the people's livelihood, public power, public policy response, system and platform construction).

\section{Results Analysis}

Public affairs mainly refers to the work of government public, the work for the government planning or preparing for the classification of the public, the work content and process to the public, any citizen can carry out query and supervision through specific ways, such as open data, open network and so on.

\subsection{The evaluation of open government about provincial government departments}

The average score of provincial government departments is 72.1844 , the distribution is generally normal distribution, the overall work of open government is in the steady progress stage. The general index average score index was $71.56 \%$, the average score of special index was $76.64 \%$. The general indicators score index of Provincial Economic and Information Commission, the Provincial Department of culture, food and Drug Supervision Bureau, the provincial Environmental Protection Bureau and the Provincial Quality Supervision Bureau of is all higher than 80\%; for the special indicators, the special indicators score index of the provincial food and drug administration, the Provincial Work Safety Supervision Bureau, the Provincial Department of culture, the provincial Price Bureau, the provincial human resources and social security department Province, Industrial and Commercial Bureau score index reached more than 90\%. In the 42 provincial government departments of this evaluation, the Provincial Department of culture ranked first, provincial economic and Information Commission, the provincial food and Drug Supervision Bureau, the Provincial Quality Supervision Bureau, the provincial Environmental Protection Bureau, the provincial SASAC, the provincial development and Reform Commission, the provincial forestry department, the provincial Industrial and Commercial Bureau and the Provincial Department of human resources and social security in second to tenth.

\subsection{The evaluation of open government about municipal government departments}

From the score of the indicators, the government of the 17 cities at the corresponding level of government affairs works well (as shown in figure 1). The average assessment score of the 34 county-level government openness is 61.12, basically reached the passing standard, but the county government level of public affairs is uneven, polarization is serious. Among them, the score index of Weihai City and Weifang City in "administrative power operation" reached $94.83 \%$ and $89.17 \%$, ahead of other municipal government; as for "information openness in key fields", Weifang City, Jinan City, Weihai city and Dezhou City, the performance is more prominent; the score index of Weihai City, Dezhou city and Weifang city in "government involvement and response concerns" is higher than 88.30\%; Weihai City, Zaozhuang City, Qingdao City and Tai'an City have a high level in "capacity building of open government", the score index reached 95\% or more. Of the 17 municipal governments evaluated, Weihai ranked first, Weifang, Dezhou, Tai'an and Linyi ranked second to fifth. 


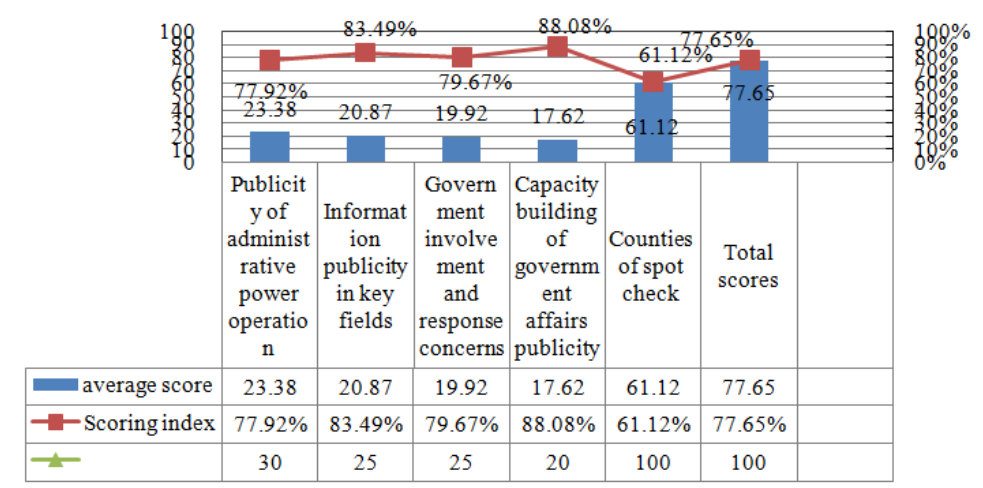

Fig. 1 Average assessment of municipal government departments

\section{Main challenge of open government}

Through the analysis of the evaluation results above, this study finds that the following problems exist in the open government of SD.

\subsection{Collection and practical applications of public opinion need to be improved}

Most units can carry out online opinions collection and public opinion surveys center on major decisions, draft of normative documents and concerns of the public. However, after the end of the consultation, $71 \%$ of the provincial government departments and $70 \%$ of the municipal government were unable to timely publish the situation of opinion collection, the adoption and the reasons for not adopted. Some units carry out less number of activities in the government website, online survey subjects of $31 \%$ provincial government departments and $29 \%$ municipal government departments are only limited to web page revision, website satisfaction, lack of major livelihood decisions polls.

\subsection{The depth and quality of information openness in some key fields need to be strengthened}

Some of the key areas of information disclosure still have unclear open standards, content is not refined enough, for example, government investment in major construction projects are mostly news information, the elements of information content is incomplete. In the field of livelihood information, some units adopt domain classification, which makes it very inconvenient for the public to obtain information. Part of the city's key areas of information disclosure directory directly linked to the relevant departments of the city's home page, it is not conducive to inquire required information, the depth and quality of openness needs to be strengthened [4].

\subsection{The interpretation of local policy is not adequate}

In the interpretation of local policy, some units still have the problem that only use a single form, lack of digital, Q\&A, charts, illustrations, audio, video and other means. There are policy interpretation column of some units, mainly reproduced by the relevant departments of the state or higher authorities, lack of the interpretation of the policy in the region. The interpretation of the information issued by the provincial government departments, which is closely related to the macro economy and the people's livelihood, is not ideal enough.

\section{Implications for the future work of open government}

In view of the challenge discovered, this paper puts forward the following implicationss for the government affairs of SD.

\subsection{Exploring the standardization of open government in SD}

The evaluation shows that the overall level and standardization of government affairs in the county government of SD are relatively low, and the development is uneven and polarization is serious. It is recommended that the provincial government office and the Provincial Quality Supervision Bureau should in the province to take the lead in promoting standardization of government affairs, promoting the depth of integration of government affairs and standardization work. Selecting a county (city, district) as a pilot to explore the way to the openness of basic characteristics, and gradually improve our province public affairs of local standards, provide the basic contents and methods of public work 
to follow for all the levels of government. On the basis of the first trial, we should strive for 5 counties (cities, districts) of our province to become standardized pilot of standardized government affairs at the national level [5].

\subsection{Re-planning provincial government information disclosure platform}

The provincial government public information platform has been used as a unified platform for the integration of provincial government portal and for the government to release the information in various regions and departments. In the comprehensive promotion of public affairs under the new situation, its development needs to be re-planned. It is recommended that the provincial government office and public affairs departments should organize experts and representatives of the public to do some research and demonstration on the future development direction of the provincial government information disclosure platform, make reasonable planning, clear its role and position. Establishing the joint update mechanism of provincial government information disclosure platform and various departments and regional portal information disclosure column, to achieve the "one point of release, multi point sharing", and solve the problem of untimely artificial regeneration which lead to inconsistent information.

\subsection{Further strengthening policy interpretation}

It is suggested that the general office of the provincial government should introduce policies and interpret relevant policies or standards, making clear provisions for the scope, time limit, form and content of the departments at various levels, so as to further standardize the interpretation of policies. To establish the synchronization mechanism of policy interpretation and policy formulation work, policy documents and interpretation program, interpretation of materials should be synchronized organization, synchronization signing and synchronous deployment.

\section{Conclusions}

Open government plays an important role in promoting the transformation of government functions, improving administrative efficiency, preventing and controlling corruption. With the analisis of the survey conducted in Shandong China, some challenges were found and implications were given to other governments considering to open.

\section{References}

[1] Janssen, K. (2011). The influence of the psi directive on open government data: an overview of recent developments. Government Information Quarterly, 28(4), 446-456.

[2] Veljković, N., Bogdanović-Dinić, S., \& Stoimenov, L. (2014). Benchmarking open government: an open data perspective. Government Information Quarterly, 31(2), 278-290.

[3] Bates, J. (2014). The strategic importance of information policy for the contemporary neoliberal state: the case of open government data in the united kingdom. Government Information Quarterly, 31(3), 388-395.

[4] Whitmore, A. (2014). Using open government data to predict war: a case study of data and systems challenges. Government Information Quarterly, 31(4), 622-630.

[5] Luna-Reyes, L. F., Bertot, J. C., \& Mellouli, S. (2014). Open government, open data and digital government. Government Information Quarterly, 31(1), 4-5. 